PROCEEDINGS OF THE

AMERICAN MATHEMATICAL SOCIETY

Volume 136, Number 6, June 2008, Pages 2091-2101

S 0002-9939(08)09181-8

Article electronically published on February 14, 2008

\title{
SPECTRAL MAPPING THEOREM FOR LINEAR HYPERBOLIC SYSTEMS
}

\author{
MARK LICHTNER \\ (Communicated by Carmen C. Chicone)
}

\begin{abstract}
We show high frequency resolvent and spectral estimates and prove the spectral mapping theorem for linear hyperbolic systems in one space dimension.
\end{abstract}

\section{INTRODUCTION}

As is well known stability and dichotomy of a linear system of ODEs

$$
\dot{z}=A z
$$

are determined by the spectrum $\sigma(A)$ of the matrix $A$. For infinite dimensional evolution equations the issue is more complex. Let $A$ be an infinitesimal generator of a $C_{0}$ semigroup $e^{A t}$. We say that $A$ has the Spectral Mapping Property (SMP) if

$$
\text { (SMP) } \sigma\left(e^{A t}\right) \backslash\{0\}=\overline{e^{\sigma(A) t}} \backslash\{0\} \quad \text { for } t \geq 0 .
$$

It is well known that (SMP) does not hold, in general, if $A$ is a generator of a $C_{0}$ semigroup. Several counterexamples are known for abstract $C_{0}$ semigroups 1, 2]. Renardy gave a more applied counterexample [3]: He constructed a lower order derivative perturbation of a two dimensional wave equation, where the growth bound $\omega(A):=\inf \left\{\omega \in \mathbb{R} \mid \exists M=M(\omega) \forall t \geq 0:\left\|e^{A t}\right\| \leq M e^{\omega t}\right\}$ is strictly larger than the spectral bound $s(A):=\sup \{\mathfrak{R e} \lambda \mid \lambda \in \sigma(A)\}$. One has the following formula for $\omega$ [1]: For each $t_{0}>0, \omega(A)=t_{0}^{-1} \log r\left(e^{A t_{0}}\right)=\lim _{t \rightarrow \infty} t^{-1} \log \left\|e^{A t}\right\|$, where $r\left(e^{A t_{0}}\right)$ denotes the spectral radius of $e^{A t_{0}}$. Hence (SMP) contains spectrum determined growth $\omega(A)=s(A)$. Renardys counterexample not only illustrates that (SMP) is violated, in general, for linear hyperbolic PDEs in higher dimensions (see also 4), moreover it destroys hopes that "applied" problems are somehow well behaved. This is annoying since computing spectra of linearizations is the most widely used practical method for assessing stability and bifurcations of physical systems. It is known that for $C_{0}$ semigroups in Hilbert space the set of counterexamples, where $\omega(A)>s(A)$, is small in the sense of the Baire category [2].

Received by the editors March 13, 2007.

2000 Mathematics Subject Classification. Primary 47D03, 47D06, 34D09, 35P20; Secondary 37L10, 37D10.

Key words and phrases. Linear hyperbolic systems, estimates for spectrum and resolvent, spectral mapping theorem, $C_{0}$ semigroups, exponential dichotomy, invariant manifolds.

This work has been supported by DFG Research Center MATHEON, 'Mathematics for key technologies' in Berlin.

(C)2008 American Mathematical Society Reverts to public domain 28 years from publication 
Moreover, (SMP) implies existence of dichotomic projections for (1.1) under the presence of a spectral gap for $A$. Exponential dichotomies are required for the proof of center manifold theorems for nonlinear evolution equations, which are often applied to analyse the local dynamics and bifurcations of (infinite dimensional) dynamical systems. The theory is, to our knowledge, restricted to classes of evolution equations where (SMP) is known, which includes ODEs and large classes of DDEs and semilinear parabolic PDEs. The unavailability/failure of (SMP) has been a main obstacle for extending the theory [6, 7, 8, 5] to hyperbolic PDEs. Quite recently (SMP) has been proven for Schrödinger equations 9 .

Our main result is that (SMP) holds true for a general class of linear hyperbolic systems in one space dimension. We prove (SMP) by establishing high frequency estimates for spectra and resolvents in terms of reduced (block)diagonal systems and applying the Gearhart-Prüss spectral mapping theorem [10, 11] (see Theorem 4.2). Related results can be found in [12,13, but precise resolvent and spectral estimates as well as the spectral mapping property (SMP) are missing. Our estimates can be used even in cases where the Gearhart-Prüss theorem does not apply. This has important applications for nonlinear hyperbolic systems, see [14, 15, 16, 17, which often appear in applied problems.

\section{Results}

We consider linear hyperbolic systems in the following normal form:

$$
\left\{\begin{array}{l}
\frac{\partial}{\partial t}\left(\begin{array}{l}
u(t, x) \\
v(t, x)
\end{array}\right)+K(x) \frac{\partial}{\partial x}\left(\begin{array}{c}
u(t, x) \\
v(t, x)
\end{array}\right)+C(x)\left(\begin{array}{c}
u(t, x) \\
v(t, x)
\end{array}\right)=0 \\
\frac{d}{d t}[v(t, l)-D u(t, l)]=F u(t, \cdot)+G v(t, \cdot), \\
u(t, 0)=E v(t, 0) .
\end{array}\right.
$$

The assumptions are:

(HI) $K$ is a blockdiagonal $n \times n$ matrix of the form

$$
K=\text { blockdiag }\left(k_{i} I_{d_{i}}\right)_{1 \leq i \leq \alpha+\beta},
$$

where $d_{i} \in \mathbb{N}, d_{i}>0, \alpha \in \mathbb{N}, \beta \in \mathbb{N}, \sum_{i=1}^{\alpha} d_{i}=n_{1}, \sum_{i=1}^{\beta} d_{\alpha+i}=n_{2}$, $I_{d_{i}}$ denotes the identity matrix in $\mathbb{C}^{d_{i} \times d_{i}}$ and $k_{i} \in C^{1}([0, l], \mathbb{R})$ satisfy for $x \in[0, l]$

$$
k_{i}(x)>0,1 \leq i \leq \alpha, \quad k_{j}(x)<0, \alpha+1 \leq j \leq \alpha+\beta .
$$

(HII) (HII) $C(x)=\left(C_{i j}(x)\right)_{1 \leq i, j \leq \alpha+\beta} \in \mathbb{C}^{n \times n}$ with $C_{i j}(x) \in \mathbb{C}^{d_{i} \times d_{j}}$ and

$C_{i i} \in L^{\infty}(] 0, l\left[, \mathbb{C}^{d_{i} \times d_{i}}\right), C_{i j} \in \mathrm{BV}\left([0, l], \mathbb{C}^{d_{i} \times d_{j}}\right), 1 \leq i, j \leq \alpha+\beta, i \neq j$.

(HIII) If $i \neq j$ and $k_{i}(x)=k_{j}(x)$ for some $x \in[0, l]$, then $C_{i j}$ vanishes on $[0, l]$.

(HIV) $u(t, x)=\left(u_{i}(t, x)\right)_{1 \leq i \leq n_{1}} \in \mathbb{C}^{n_{1}}, v(t, x)=\left(v_{i}(t, x)\right)_{1 \leq i \leq n_{2}} \in \mathbb{C}^{n_{2}}$.

(HV) $D \in \mathbb{C}^{n_{2} \times n_{1}}, E \in \mathbb{C}^{n_{1} \times n_{2}}$ and

$$
F: C\left([0, l], \mathbb{C}^{n_{1}}\right) \rightarrow \mathbb{C}^{n_{2}}, \quad G: C\left([0, l], \mathbb{C}^{n_{2}}\right) \rightarrow \mathbb{C}^{n_{2}}
$$

are linear continuous operators. 
Let

$$
A\left(\begin{array}{l}
u \\
v \\
d
\end{array}\right):=\left(\begin{array}{c}
-K \frac{\partial}{\partial x}\left(\begin{array}{l}
u \\
v
\end{array}\right)-C\left(\begin{array}{l}
u \\
v
\end{array}\right) \\
F u+G v
\end{array}\right)
$$

denote the infinitesimal generator for $(\mathrm{H})$ with domain

$$
\mathcal{D}(A):=\left\{(u, v, d) \in W^{1,2}(] 0, l\left[, \mathbb{C}^{n}\right) \times \mathbb{C}^{n_{2}} \mid u(0)=E v(0), d=v(l)-D u(l)\right\} .
$$

Then $A$ generates a $C_{0}$ semigroup $e^{A t}$ on the Hilbert space $L^{2}(] 0, l\left[, \mathbb{C}^{n}\right) \times \mathbb{C}^{n_{2}}$ (see [18, 19, 12]). Let $R(\lambda, A)=(\lambda I-A)^{-1}$ denote resolvent, $\sigma(A)$ spectrum, $\sigma_{p}(A)$ pointspectrum and let $\rho(A)=\mathbb{C} \backslash \sigma(A)$ be the resolvent set of $A$. Our main result is

Theorem 2.1 (Spectral Mapping Theorem).

$$
\sigma\left(e^{A t}\right) \backslash\{0\}=\overline{e^{\sigma(A) t}} \backslash\{0\} \quad \text { for } \quad t \geq 0 .
$$

In Theorem $2.1 \overline{e^{\sigma(A) t}}$ denotes the closure of the set $e^{\sigma(A) t}$. We prove (SMP) by establishing high frequency estimates for spectra and resolvents of $A$ in terms of reduced (block)diagonal systems: Let $C_{b 0}$ be the block diagonal matrix containing the square matrices $C_{i i}$

$$
C_{b 0}:=\text { blockdiag }\left(C_{i i}\right)_{1 \leq i \leq \alpha+\beta} .
$$

Then the reduced system is per definitionem given by

$$
\left\{\begin{array}{l}
\frac{\partial}{\partial t}\left(\begin{array}{l}
u(t, x) \\
v(t, x)
\end{array}\right)+K(x) \frac{\partial}{\partial x}\left(\begin{array}{l}
u(t, x) \\
v(t, x)
\end{array}\right)+C_{b 0}(x)\left(\begin{array}{l}
u(t, x) \\
v(t, x)
\end{array}\right)=0, \\
u(t, 0)=E v(t, 0), \quad v(t, l)=D u(t, l) .
\end{array}\right.
$$

We denote the infinitesimal generator to $\left(\mathrm{H}_{0}\right)$ by

$$
\begin{aligned}
A_{0}\left(\begin{array}{l}
u \\
v
\end{array}\right) & :=-K \frac{\partial}{\partial x}\left(\begin{array}{l}
u \\
v
\end{array}\right)-C_{b 0}\left(\begin{array}{l}
u \\
v
\end{array}\right), \\
\mathcal{D}\left(A_{0}\right) & :=\left\{(u, v) \in W^{1,2}(] 0, l\left[, \mathbb{C}^{n}\right) \mid u(0)=E v(0), v(l)=D u(l)\right\} .
\end{aligned}
$$

$A_{0}$ generates a $C_{0}$ semigroup on $L^{2}(] 0, l\left[, \mathbb{C}^{n}\right)$.

Lemma 2.2. The resolvent $R(\lambda, A)\left(R\left(\lambda, A_{0}\right)\right)$ is compact, and $\sigma(A)=\sigma_{p}(A)$ $\left(\sigma\left(A_{0}\right)=\sigma_{p}\left(A_{0}\right)\right)$.

Denote $\mathbb{C}_{r, d}:=\{\lambda \in \mathbb{C}|-r<\mathfrak{R e} \lambda<r,| \mathfrak{I m} \lambda \mid>d\}, \quad \mathbb{C}_{r}:=\mathbb{C}_{r,-1}$.

Lemma 2.3 (Estimates for spectrum). There exists an exponential polynomial $h_{0}$ and an entire function $h$ with 1

- $\sigma(A)=\{\lambda \in \mathbb{C} \mid h(\lambda)=0\}, \sigma\left(A_{0}\right)=\left\{\lambda \in \mathbb{C} \mid h_{0}(\lambda)=0\right\}$.

- Put $\tilde{h}(\lambda):=\lambda^{-n} h(\lambda)$ :

$$
\forall r>0 \exists c, d>0 \forall \lambda \in \mathbb{C}_{r, d}: \quad\left|\tilde{h}(\lambda)-h_{0}(\lambda)\right| \leq c \frac{1}{|\lambda|} .
$$

If $A_{0}$ has a nonempty spectrum define $\gamma_{-}:=\inf \left\{\mathfrak{R e} \lambda \mid h_{0}(\lambda)=0\right\}$ and $\gamma_{+}:=$ $\sup \left\{\mathfrak{R e} \lambda \mid h_{0}(\lambda)=0\right\}$. Since $h_{0}$ is an exponential polynomial, $\gamma_{-}$and $\gamma_{+}$are finite. If $A_{0}$ has an empty spectrum put $\gamma_{+}:=-\infty$ and $\gamma_{-}:=\infty$. For $\lambda \in \mathbb{C}$ and $\epsilon>0$ let $B_{\epsilon}(\lambda):=\{z \in \mathbb{C}|| z-\lambda \mid<\epsilon\}$ denote the ball around $\lambda$ with radius $\epsilon$.

\footnotetext{
${ }^{1} h_{0}$ and $h$ are defined in formulas (3.4) and (3.6).
} 
Lemma 2.4. For each $\gamma>\gamma_{+}$there exist only finitely many eigenvalues $\lambda$ of $A$ that satisfy $\mathfrak{R e} \lambda \geq \gamma$.

Lemma 2.5. Suppose $A_{0}$ has nonempty spectrum. Then the following hold:

- For each $\delta>0$ there are only finitely many eigenvalues $\lambda$ of $A$ which satisfy $\mathfrak{R e} \lambda \leq \gamma_{-}-\delta$ or $\mathfrak{R e} \lambda \geq \gamma_{+}+\delta$.

- For $\epsilon>0$ there exists $d>0$ such that $\sigma(A) \cap\{\lambda \in \mathbb{C}|| \mathfrak{I m} \lambda \mid \geq d\} \subset$ $\bigcup_{h_{0}(\lambda)=0} B_{\epsilon}(\lambda)$.

- Suppose $\rho=\inf \left\{\left|\lambda_{1}-\lambda_{2}\right| \mid \lambda_{1} \neq \lambda_{2}, h_{0}\left(\lambda_{1}\right)=h_{0}\left(\lambda_{2}\right)=0\right\}>0$. Then for $\eta<\frac{\rho}{2}$ there exists $d>0$ such that for each $\lambda_{0} \in \mathbb{C}$ with $h_{0}\left(\lambda_{0}\right)=0$ and $\left|\mathfrak{I m} \lambda_{0}\right| \geq d$ there exists $\lambda \in B_{\eta}\left(\lambda_{0}\right)$ with $h(\lambda)=0$. Both $h$ and $h_{0}$ have the same number of zeros in each $B_{\eta}\left(\lambda_{0}\right)$ counted with multiplicities. If $A_{0}$ only possesses algebraically simple eigenvalues, then the eigenvalues $\lambda \in B_{\eta}\left(\lambda_{0}\right)$ of $A$ are unique and algebraically simple.

Lemma 2.6 (Estimates for resolvent). Let $U \subset \rho(A)$ so that $\sup _{\lambda \in U}|\mathfrak{R e} \lambda|<\infty$ and $\inf _{\lambda \in U}\left|h_{0}(\lambda)\right|>0$. Then there exists $d>0$ so that for $\lambda \in U$ and $|\Im \mathfrak{m} \lambda| \geq d$

- $R(\lambda, A)\left(\begin{array}{l}f \\ g \\ b\end{array}\right)=\left(\begin{array}{c}u \\ v \\ v(l)-D u(l)\end{array}\right),\left(\begin{array}{l}u \\ v\end{array}\right)=R\left(\lambda, A_{0}\right)\left(\begin{array}{l}f \\ g\end{array}\right)+\frac{1}{\lambda} \mathcal{E}(\lambda, A)\left(\begin{array}{l}f \\ g \\ b\end{array}\right)$.

- $R\left(\lambda, A_{0}\right)$ and $\mathcal{E}(\lambda, A)$ are bounded. In particular the resolvent $R(\lambda, A)$ is bounded on $U$.

- $\mathcal{E}(\lambda, A)$ can be represented as a series $\sum_{k=0}^{\infty} \lambda^{-k} R_{k}(\lambda, A)$, where for each $R_{k}$ there exists a closed formula in terms of integrals of elementary functions of $\lambda$.

Remark 2.7. For all applications we know that conditions (HI)-(HV) are fulfilled. Our conditions are slightly more general than [12]; we include equal speed systems which are required for applications to semiconductor laser dynamics [14, 17.

\section{Estimates FOR SPECTRA AND RESOLVENTS}

Consider (H) and assume (HI)-(HV). We prove Lemmas 2.2.2.6.

Let $T$ denote the fundamental matrix satisfying

$$
\begin{array}{rlr}
\frac{d}{d x} T(x, y, \lambda) & =-K(x)^{-1}(\lambda I+C(x)) T(x, y, \lambda) \quad \text { for } x, y \in[0, l], \\
T(y, y, \lambda) & =I \quad \text { for } y \in[0, l],
\end{array}
$$

and $T_{0}$ the fundamental system for

$$
\begin{array}{rlr}
\frac{d}{d x} T_{0}(x, y, \lambda) & =-K^{-1}(x)\left(\lambda I+C_{b 0}(x)\right) T_{0}(x, y, \lambda) \quad \text { for } x, y \in[0, l] \\
T_{0}(y, y, \lambda) & =I \quad \text { for } y \in[0, l] .
\end{array}
$$

For $1 \leq i \leq \alpha+\beta$ there exist $F_{i}$ depending only on $C_{b 0}$ and $K, F_{i}:[0, l]^{2} \rightarrow \mathbb{C}^{d_{i} \times d_{i}}$, $F_{i}(\cdot, y) \in W^{1, \infty}\left([0, l], \mathbb{C}^{d_{i} \times d_{i}}\right)$ for $y \in[0, l]$, so that for $F:=\left(\operatorname{blockdiag} F_{i}\right)_{1 \leq i \leq \alpha+\beta}$ we have

$$
T_{0}(x, y, \lambda)=\exp \left(-\lambda \int_{y}^{x} K^{-1}(z) d z\right) F(x, y) .
$$


Moreover for $x \geq y \geq z$ we have $F_{i}(x, z)=F_{i}(x, y) F_{i}(y, z)$. Indeed: Define $F_{i}$ to be the solution of

$$
\frac{d}{d x} F_{i}(x, y)=-k_{i}^{-1}(x) C_{i i}(x) F_{i}(x, y), \quad F_{i}(y, y)=I_{d_{i}} .
$$

From (2.1) and (HI) it follows that $K, C_{b 0}$ and $\exp \left(-\lambda \int_{y}^{x} K^{-1}(z) d z\right)$ commute. Hence the right hand side of (3.3) solves (3.2).

We have $\lambda \in \sigma_{p}(A)$ iff there exists $v_{0} \in \mathbb{C}^{n_{2}}, v_{0} \neq 0$, such that

$$
\left(\begin{array}{l}
u \\
v
\end{array}\right)(x)=T(x, 0, \lambda)\left(\begin{array}{c}
E \\
I
\end{array}\right) v_{0} \quad \text { and } \quad\left(-\lambda D \delta_{l}-F, \lambda I \delta_{l}-G\right)\left(\begin{array}{l}
u \\
v
\end{array}\right)=0 .
$$

Hence we define

$$
\begin{aligned}
H(\lambda) & :=\left(-\lambda D \delta_{l}-F, \lambda I \delta_{l}-G\right) T(\cdot, 0, \lambda)\left(\begin{array}{c}
E \\
I
\end{array}\right) \quad\left(\in \mathbb{C}^{n_{2} \times n_{2}}\right) \\
h(\lambda) & :=\operatorname{det} H(\lambda) .
\end{aligned}
$$

Using this notation we have

$$
\sigma_{p}(A)=\{\lambda \in \mathbb{C} \mid h(\lambda)=0\} .
$$

We call $h(\lambda)$ the characteristic function and $H(\lambda)$ the characteristic matrix to $(\mathrm{H})$. For $\lambda \in \sigma\left(A_{p}\right)$ the eigenspace can be represented as

$$
\operatorname{Eig}(A, \lambda)=\left\{\left(\begin{array}{c}
u \\
v \\
v(l)-D u(l)
\end{array}\right) \mid\left(\begin{array}{l}
u(\cdot) \\
v(\cdot)
\end{array}\right)=T(\cdot, 0, \lambda)\left(\begin{array}{c}
E \\
I
\end{array}\right) v_{0}, \quad v_{0} \in \operatorname{Ker} H(\lambda)\right\} .
$$

The geometric multiplicity of each eigenvalue is less than or equal to $n_{2}$.

The resolvent equation $R(\lambda, A)(f, g, b)=(u, v, d)$ for $(u, v, d) \in \mathcal{D}(A)$ and $(f, g, b)$ $\in L^{2}(] 0, l\left[, \mathbb{C}^{n}\right) \times \mathbb{C}^{n_{2}}$ reads

$$
K \frac{\partial}{\partial x}\left(\begin{array}{l}
u \\
v
\end{array}\right)+(\lambda I+C)\left(\begin{array}{l}
u \\
v
\end{array}\right)=\left(\begin{array}{l}
f \\
g
\end{array}\right), \quad\left(-\lambda D \delta_{l}-F, \lambda I \delta_{l}-G\right)\left(\begin{array}{l}
u \\
v
\end{array}\right)=b .
$$

This is equivalent to

$$
\begin{aligned}
\left(\begin{array}{l}
u \\
v
\end{array}\right)(x) & =T(x, 0, \lambda)\left(\begin{array}{c}
E \\
I
\end{array}\right) v(0)+\int_{0}^{x} T(x, y, \lambda) K(y)^{-1}\left(\begin{array}{l}
f \\
g
\end{array}\right)(y) d y, \\
b & =\left(-\lambda D \delta_{l}-F, \lambda I \delta_{l}-G\right)\left(\begin{array}{l}
u \\
v
\end{array}\right) .
\end{aligned}
$$

If $\lambda \notin \sigma_{p}(A)$, by inserting the first equation into the second one, we get that the resolvent equation has a unique solution (hence $\sigma_{p}(A)=\sigma(A)$ ) and $v(0)=$ $H(\lambda)^{-1} \beta(\lambda)(f, g, b)$, where

$$
\beta(\lambda)(f, g, b):=b+\left(\lambda D \delta_{l}+F, G-\lambda I \delta_{l}\right) \int_{0}^{\cdot} T(\cdot, y, \lambda) K(y)^{-1}\left(\begin{array}{c}
f(y) \\
g(y)
\end{array}\right) d y .
$$

Thus we have:

Proposition 3.1. For any $\lambda$ such that $h(\lambda) \neq 0$ the resolvent $R(\lambda, A)=(\lambda I-A)^{-1}$ is given by

$$
\left[R(\lambda, A)\left(\begin{array}{l}
f \\
g \\
b
\end{array}\right)\right](x)=\left(\begin{array}{c}
u(x) \\
v(x) \\
v(l)-D u(l)
\end{array}\right)
$$


where

$$
\left(\begin{array}{l}
u \\
v
\end{array}\right)=T(\cdot, 0, \lambda)\left(\begin{array}{c}
E \\
I
\end{array}\right) H(\lambda)^{-1} \beta(\lambda)(f, g, b)+\int_{0}^{\cdot} T(\cdot, y, \lambda) K(y)^{-1}\left(\begin{array}{c}
f(y) \\
g(y)
\end{array}\right) d y .
$$

From Proposition 3.1 we get Lemma 2.2

Remark 3.2. $\lambda I-A$ is a Fredholm operator of index zero.

Denote

$$
h_{0}(\lambda):=\operatorname{det} H_{0}(\lambda), \quad H_{0}(\lambda):=(-D, I) T_{0}(l, 0, \lambda)\left(\begin{array}{c}
E \\
I
\end{array}\right) \quad\left(\in \mathbb{C}^{n_{2} \times n_{2}}\right) .
$$

$h_{0}$ is called a characteristic function and $H_{0}$ a characteristic matrix to the reduced system $\left(\mathrm{H}_{0}\right)$.

For any $\lambda$ such that $h_{0}(\lambda) \neq 0$ we have the following explicit formula for the resolvent $R\left(\lambda, A_{0}\right)=\left(\lambda I-A_{0}\right)^{-1}$ :

$$
\begin{aligned}
R\left(\lambda, A_{0}\right)\left(\begin{array}{c}
f \\
g
\end{array}\right)= & T_{0}(\cdot, 0, \lambda)\left(\begin{array}{c}
E \\
I
\end{array}\right) H_{0}(\lambda)^{-1} \beta_{0}(\lambda)(f, g) \\
& +\int_{0} T_{0}(\cdot, y, \lambda) K(y)^{-1}\left(\begin{array}{l}
f(y) \\
g(y)
\end{array}\right) d y,
\end{aligned}
$$

where

$$
\beta_{0}(\lambda)(f, g):=(D,-I) \int_{0}^{l} T_{0}(l, y, \lambda) K(y)^{-1}\left(\begin{array}{c}
f(y) \\
g(y)
\end{array}\right) d y .
$$

Define the coupling matrix $C_{1}(x):=C(x)-C_{b 0}(x)$ and for $k \geq 1$

$$
T_{k}(x, y, \lambda):=-\lambda T_{0}(x, y, \lambda) \int_{y}^{x} T_{0}(y, z, \lambda) K^{-1}(z) C_{1}(z) T_{k-1}(z, y, \lambda) d z .
$$

Each $T_{k}, k \geq 1$, satisfies the initial value problem

$$
\frac{d}{d x} T_{k}(x, y, \lambda)=-K^{-1}(x)\left(\lambda I+C_{b 0}(x)\right) T_{k}(x, y, \lambda)-\lambda K^{-1}(x) C_{1}(x) T_{k-1}(x, y, \lambda)
$$

with $T_{k}(x, x, \lambda)=0$, and can be calculated recursively in terms of integrals of elementary functions and $F$. The only unknown $F$ does not depend on $\lambda$. We will see that the series $\sum_{k=0}^{\infty} \lambda^{-k} T_{k}(x, y, \lambda)$ converges in $W^{1, \infty}$ for sufficiently large $|\mathfrak{I m} \lambda|$ to the fundamental matrix $T$ of (3.1). However, $T_{k}, k \geq 2$, are not bounded for $\lambda$ chosen from any stripe $\mathbb{C}_{r}$. Indeed we will see in the following that the expressions $T_{k}, k \geq 2$, contain some power terms $\lambda^{i}$ with $i$ up to the lower integer part of $k / 2$ which will be due to successive failures in partial integration in the formula (3.7). After reordering terms for any finite $\kappa \in \mathbb{N}$ we will obtain an explicit representation of the form

$$
T(x, y, \lambda)=\sum_{k=0}^{\kappa} \lambda^{-k} F_{k}(x, y, \lambda)+O\left(\lambda^{-(\kappa+1)}\right),
$$

for $\lambda$ in a stripe $\mathbb{C}_{r}$ and sufficiently large $|\mathfrak{I m} \lambda|$, where each $F_{k}$ is of order 1 with respect to $\lambda$ on $\mathbb{C}_{r}$. To see this we calculate the first two steps, $T_{1}$ and $T_{2}$. Put

$$
f_{0}(x, y, \lambda):=T_{0}(x, y, \lambda)\left(y_{0}^{(1)}, \ldots, y_{0}^{(\alpha+\beta)}\right)^{t}
$$

with the arbitrary fixed initial data $y_{0}^{(i)} \in \mathbb{C}^{d_{i}}, 1 \leq i \leq \alpha+\beta$. Define

$$
f_{k}:=-\lambda \int_{y}^{x} T_{0}(x, z, \lambda) K^{-1}(z) C_{1}(z) f_{k-1}(z, y, \lambda) d z \text { for } k \geq 1 .
$$


Then according to (3.3), the $i$-th component $1 \leq i \leq \alpha+\beta$ of $f_{k}$ is

$$
\begin{aligned}
f_{0}^{(i)}(x, y, \lambda)= & \exp \left(-\int_{y}^{x} \lambda k_{i}^{-1}(u) d u\right) F_{i}(x, y) y_{0}^{(i)}, \\
f_{k}^{(i)}(x, y, \lambda)= & -\lambda \exp \left(-\int_{y}^{x} \lambda k_{i}^{-1}(u) d u\right) F_{i}(x, y) \\
& \sum_{\substack{1 \leq l \leq \alpha+\beta \\
l \neq i}} \int_{y}^{x} \exp \left(\lambda \int_{y}^{z} k_{i}^{-1}(u) d u\right) F_{i}(y, z) \frac{C_{i l}(z)}{k_{i}(z)} f_{k-1}^{(l)}(z, y, \lambda) d z .
\end{aligned}
$$

By (HII) and (HIII) we can perform partial integration and get rid of the $\lambda$ factor appearing in the recursion formula for $f_{k}^{(i)}$ : Denote $\kappa_{i l}(x):=k_{i}^{-1}(x)-k_{l}^{-1}(x)$, then

$$
\begin{aligned}
f_{1}^{(i)}(x, y, \lambda)=-\exp \left(-\lambda \int_{y}^{x} k_{i}^{-1}(u) d u\right) F_{i}(x, y) \\
\quad \times \sum_{\substack{1 \leq l \leq \alpha+\beta \\
l \neq i}} \int_{y}^{x} \lambda \kappa_{i l}(z) \exp \left(\int_{y}^{z} \lambda \kappa_{i l}(u)\right) F_{i}(y, z) \frac{C_{i l}(z)}{k_{i}(z)} \frac{F_{l}(z, y)}{\kappa_{i l}(z)} y_{0}^{(l)} d z \\
=\sum_{\substack{1 \leq l \leq \alpha+\beta \\
l \neq i}}\left\{-\exp \left(-\lambda \int_{y}^{x} k_{l}^{-1}(u) d u\right) \frac{C_{i l}(x)}{k_{i}(x)} \frac{F_{l}(x, y)}{\kappa_{i l}(x)}\right. \\
\quad+\exp \left(-\int_{y}^{x} \lambda k_{i}^{-1}(u) d u\right) F_{i}(x, y) \\
\left.\quad \times\left[\frac{C_{i l}(y)}{k_{i}(y)} \frac{1}{\kappa_{i l}(y)}+\int_{y}^{x} \exp \left(\int_{y}^{z} \lambda \kappa_{i l}(u) d u\right) \frac{d}{d z}\left(F_{i}(y, z) \frac{C_{i l}(z)}{k_{i}(z)} \frac{F_{l}(z, y)}{\kappa_{i l}(z)}\right) d z\right]\right\} y_{0}^{(l)} .
\end{aligned}
$$

Note that for partial integration we used the fact that in the sum for $l \neq i$ in the formula for $f_{1}^{(i)}$ the leading $\lambda$-exponential of $f_{0}^{(l)}$ is $e^{-\int_{y}^{x} \lambda k_{l}^{-1}(u) d u}$. However, now $f_{1}^{(i)}$ not only contains $2(\alpha+\beta-1)$ terms with $\lambda$-exponential $e^{-\int_{y}^{x} \lambda k_{i}^{-1}(u) d u}$ but also $(\alpha+\beta-1)$ terms of the form $e^{-\int_{y}^{x} \lambda k_{l}^{-1}(u) d u}, 1 \leq l \leq \alpha+\beta, l \neq i$. Therefore, in the next step for $f_{2}$ we are not able to get rid of all $\lambda$ terms by partial integration as in the first step:

$$
\begin{aligned}
& f_{2}^{(i)}(x, y, \lambda)=-\exp \left(-\lambda \int_{y}^{x} k_{i}^{-1}(u) d u\right) F_{i}(x, y) \sum_{\substack{1 \leq l_{2}, l_{1} \leq \alpha+\beta \\
l_{2} \neq i, l_{1} \neq l_{2}}} \lambda \\
& \times \int_{y}^{x}\left\{-\exp \left(\int_{y}^{z_{2}} \lambda \kappa_{i l_{1}}(u) d u\right) F_{i}(y, z) \frac{C_{i l_{2}}\left(z_{2}\right) C_{l_{2} l_{1}}\left(z_{2}\right)}{k_{i}\left(z_{2}\right) k_{l_{2}}\left(z_{2}\right)} \frac{F_{l_{1}}\left(z_{2}, y\right)}{\kappa_{l_{2} l_{1}}\left(z_{2}\right)}\right. \\
& +\exp \left(\lambda \int_{y}^{z_{2}} \kappa_{i l_{2}}(u) d u\right) F_{i}\left(y, z_{2}\right) \frac{C_{i l_{2}}\left(z_{2}\right)}{k_{i}\left(z_{2}\right)} F_{l_{2}}\left(z_{2}, y\right)\left[\frac{C_{l_{2} l_{1}}(y)}{k_{l_{2}}(y)} \frac{1}{\kappa_{l_{2} l_{1}}(y)}\right. \\
& \left.\left.+\int_{y}^{z_{2}} \exp \left(\lambda \int_{y}^{z_{1}} \kappa_{l_{2} l_{1}}(u) d u\right) \frac{d}{d z_{1}}\left(F_{l_{2}}\left(y, z_{1}\right) \frac{C_{l_{2} l_{1}}\left(z_{1}\right)}{k_{l_{2}}\left(z_{1}\right)} \frac{F_{l_{1}}\left(z_{1}, y\right)}{\kappa_{l_{2} l_{1}}\left(z_{1}\right)}\right) d z_{1}\right]\right\} y_{0}^{\left(l_{1}\right)} d z_{2} .
\end{aligned}
$$


Partial integration is not possible for the terms in the sum corresponding to $l_{1}=i$. Therefore we are forced to keep $(\alpha+\beta-1)$ terms containing $\lambda$ factors:

$$
\begin{aligned}
f_{2}^{(i)}(x, y, \lambda)= & -\lambda \exp \left(-\lambda \int_{y}^{x} k_{i}^{-1}(u) d u\right) F_{i}(x, y) \\
& \times \sum_{\substack{1 \leq l_{2} \leq \alpha+\beta \\
l_{2} \neq i}} \int_{y}^{x} \frac{C_{i l_{2}}\left(z_{2}\right) C_{l_{2} i}\left(z_{2}\right)}{k_{i}\left(z_{2}\right) k_{l_{2}}\left(z_{2}\right)} \frac{F_{i}\left(z_{2}, y\right)}{\kappa_{l_{2} i}\left(z_{2}\right)} d z_{2} \cdot y_{0}^{(i)} \\
& + \text { terms of order } 1 .
\end{aligned}
$$

However, in the next (third) step for these $(\alpha+\beta-1)$ terms containing a $\lambda$ factor partial integration can be done, so that in the third step there will be no $\lambda^{2}$ factors, only $\lambda$ or 1 factors. Factors with $\lambda^{2}$ in the multisums will first appear in the fourth step. Thus, generally for $m \in \mathbb{N}$, terms containing $\lambda^{m}$ factors appear for the first time in the $(2 m)$-th recursion step. Besides these $\lambda^{m}$ terms there only appear terms which are bounded for $\lambda \in \mathbb{C}_{r}$. After reordering terms we have proven the following

Lemma 3.3. There exists a sequence $F_{k}(x, y, \lambda)$ of matrices with

- $F_{0}=T_{0}$,

- $\forall r>0 \exists c>0 \forall \lambda \in \mathbb{C}_{r},(x, y) \in[0, l]^{2}, k \in \mathbb{N}:\left\|F_{k}(x, y, \lambda)\right\| \leq c^{k}$.

- For $r>0$ there exists $d>0$ such that for $\lambda \in \mathbb{C}_{r, d}$ the series $\sum_{k=0}^{\infty} \lambda^{-k} F_{k}(x, y, \lambda)$ converges absolutely $\left(\right.$ in $\left.L^{\infty}\left([0, l] \times[0, l], \mathbb{C}^{n \times n}\right)\right)$ to $T(x, y, \lambda)$.

- $\forall r>0 \exists c, d>0 \forall \lambda \in \mathbb{C}_{r, d}:\left\|T(x, y, \lambda)-T_{0}(x, y, \lambda)\right\| \leq c \frac{1}{|\lambda|}$.

Remark 3.4. Each $F_{k}$ can be calculated from $T_{n}$ for $n=1, \ldots, 2 k$.

Consequently we get:

Lemma 3.5. For $r>0$ there exist $c, d>0$ such that for $\lambda \in \mathbb{C}_{r, d}$ we have

- $\left\|\tilde{H}(\lambda)-H_{0}(\lambda)\right\| \leq c \frac{1}{|\lambda|}$, where $\tilde{H}(\lambda):=\lambda^{-1} H(\lambda)$.

- Put $\tilde{h}(\lambda):=\operatorname{det} \tilde{H}(\lambda)$. Then

$$
\left|\tilde{h}(\lambda)-h_{0}(\lambda)\right| \leq c \frac{1}{|\lambda|} .
$$

- $\left\|\tilde{H}(\lambda)^{-1}-H_{0}(\lambda)^{-1}\right\| \leq c \frac{1}{|\lambda|}$.

We need the following lemma

Lemma 3.6. Let $f$ be an exponential polynomial of the form $f(\lambda)=\sum_{j=1}^{r} a_{j} e^{b_{j} \lambda}$ $\left(\lambda, a_{j} \in \mathbb{C}, b_{j} \in \mathbb{R}\right)$. Let $Z=\{\lambda \in \mathbb{C} \mid f(\lambda)=0\}$ denote the zero set of $f$. For all $\delta>0, \alpha, \beta \in \mathbb{R}$ with $\alpha \leq \beta$ there exists a constant $m=m(\delta, \alpha, \beta)>0$ such that for all $\lambda \in \mathbb{C}$, which satisfy $\operatorname{dist}(\lambda, Z) \geq \delta, \alpha \leq \mathfrak{R e} \lambda \leq \beta$, we have $|f(\lambda)|>m(\delta, \alpha, \beta)$.

Proof. We give a correct proof of [20, Lemma 2.2]. Suppose the assertion was false. Then there exists a sequence $\left(\lambda_{m}\right)_{m \in \mathbb{N}}, \alpha \leq \mathfrak{R e} \lambda_{m} \leq \beta$, so that $f\left(\lambda_{m}\right) \rightarrow_{m \rightarrow \infty} 0$, $\operatorname{dist}\left(\lambda_{m}, Z\right) \geq \delta>0$. Put $g_{m}(\lambda):=f\left(\lambda+\lambda_{m}\right)=\sum_{j=1}^{r} a_{j} e^{b_{j} \lambda} e^{b_{j} x_{m}} e^{i b_{j} y_{m}}$, where $\lambda_{m}=: x_{m}+i y_{m}$. Then there exists a subsequence so that $g_{m}$ converges uniformly to some function $g$ on each stripe $\mathbb{C}_{r}$ for any given $r>0$. Indeed, by passing to a subsequence we can assume that $x_{m} \rightarrow x \in[\alpha, \beta]$ and $e^{i b_{j} y_{m}} \rightarrow s_{j} \in S^{1}$. Then $g_{m}$ converges uniformly to $g(\lambda)=\sum_{j=1}^{r} a_{j} e^{b_{j} \lambda} e^{b_{j} x} s_{j}$ on each $\mathbb{C}_{r}$. Let $U:=$ $\left\{\lambda \in \mathbb{C}|| \lambda \mid<\frac{\delta}{2}\right\}$. By assumption $g_{m}(\lambda) \neq 0$ for $\lambda \in U$ and $g(0)=\lim g_{m}(0)=$ 
$\lim f\left(\lambda_{m}\right)=0$. By the Hurwitz theorem $g$ must be identical to zero on $U$, and therefore on $\mathbb{C}$. Hence for all $\lambda \in \mathbb{C}$ we get that $f(\lambda)=g_{m}\left(\lambda-\lambda_{m}\right) \rightarrow_{m \rightarrow \infty} 0$, which is a contradiction to the assumption that $\operatorname{dist}\left(\lambda_{m}, Z\right) \geq \delta>0$.

We will use the following proposition [20, Proposition 2.1] on the distribution of zeros of an exponential polynomial

Proposition 3.7. Let $f$ be an exponential polynomial. Then its set $Z$ of zeros (counted with multiplicities) is a finite union of separable sets: there exist $m<\infty$ sets $Z_{i}$ with $Z=\bigcup_{i=1}^{m} Z_{i}$ and $\inf _{\lambda, \mu \in Z_{i}, \lambda \neq \mu}|\lambda-\mu|>0$.

Denote $S_{\gamma, \omega}:=\{z \in \mathbb{C} \mid \gamma \leq \mathfrak{R e} z \leq \omega\}$.

Proof of Lemma 2.4. Let $\gamma>\gamma_{+}$. Denote $\sigma_{+}:=\{\lambda \in \sigma(A) \mid \mathfrak{R e} \lambda \geq \gamma\}$. Suppose $\sigma_{+}$is infinite. Since $A$ generates a $C_{0}$ semigroup there exists an $\omega>\gamma$ so that $\sigma_{+} \subset S_{\gamma, \omega}$. For the characteristic function $h(\lambda)$ of $(\mathrm{H})$ to be analytic the infinitely many eigenvalues of $\sigma_{+}$must accumulate at infinity within the closed stripe $S_{\gamma, \omega}$. Since $\gamma>\gamma_{+}$Lemma 3.6 yields that $\inf _{\lambda \in S_{\gamma, \omega}}\left|h_{0}(\lambda)\right|>0$. Therefore for sufficiently large $d>0$ (3.8) implies that $\inf \left\{|\tilde{h}(\lambda)|\left|\lambda \in S_{\gamma, \omega},\right| \mathfrak{I m} \lambda \mid \geq d\right\}>0$. Hence, by choosing $d>0$ sufficiently large, we get a contradiction to the assumption that there exist infinitely many $\lambda \in S_{\gamma, \omega}$ with $|\mathfrak{I m} \lambda| \geq d$ and $\tilde{h}(\lambda)=\lambda^{-n} h(\lambda)=0$. Hence $\sigma_{+}$is finite.

Proof of Lemma 2.5. Because $h_{0}$ is an exponential polynomial $A_{0}$ has infinitely many eigenvalues. Let $\delta>0$ be arbitrary and fixed. For $\theta \in[0,1]$ consider the family of operators corresponding to a perturbation from the diagonal operator with $C=C_{0}$ to the nondiagonal one with $C=C_{0}+C_{1}$ :

$$
A_{\theta}(u, v, d):=\left(-K(x) \frac{\partial}{\partial_{x}}\left(\begin{array}{l}
u \\
v
\end{array}\right)-\left(C_{0}(x)+\theta C_{1}(x)\right)\left(\begin{array}{l}
u \\
v
\end{array}\right) ; \quad \theta F u(\cdot)+\theta G v(\cdot)\right) .
$$

Let $h^{\theta}(\lambda)$ denote the corresponding characteristic function. Put $\tilde{h}^{\theta}(\lambda):=\frac{1}{\lambda^{n}} h^{\theta}(\lambda)$. Note that $\tilde{h}^{0}=h_{0}$. According to Lemma 3.6 outside of balls of radius $\frac{\delta}{2}$ around each of the zeros of $h_{0}$ in the stripe $S_{\gamma_{-}-\gamma_{+}+\delta}$, the function $\left|h_{0}\right|$ has an infimum $m>0$. From (3.8) in Lemma 3.5 there exist $c, d>0$ such that for $\lambda \in S_{\gamma_{-}-\delta, \gamma_{+}+\delta}$ with $|\mathfrak{I m}(\lambda)|>d$

$$
\left|\tilde{h}^{\theta}(\lambda)-h_{0}(\lambda)\right| \leq c \frac{1}{\lambda}
$$

Therefore for $\lambda \in S_{\gamma_{-}-\delta, \gamma_{+}+\delta} \backslash \bigcup_{h_{0}\left(\lambda_{0}\right)=0} B\left(\lambda_{0}, \delta / 2\right)$ with $|\mathfrak{I m}(\lambda)|$ sufficiently large it follows from (3.9) that $\left|\tilde{h}^{\theta}(\lambda)\right| \geq m / 2>0$. This also holds true uniformly in $\theta \in[0,1]$. Starting from $\theta=0$ this shows that, as long as we increase $\theta$ up to 1 , all but finitely many zeros of $h^{\theta}(\lambda)$ must stay in a $\delta / 2$-ball of an zero of $h_{0}(\lambda)$ (apply Proposition 3.7 and choose $\delta>0$ sufficiently small). By the continuity of a finite system of zeros with respect to the perturbation parameter $\theta$ it follows that $\left\{\lambda \in \mathbb{C} \mid \mathfrak{R e} \lambda \leq \gamma_{-}-\delta\right\} \cup\left\{\lambda \in \mathbb{C} \mid \mathfrak{R e} \lambda \geq \gamma_{+}+\delta\right\}$ contains only finitely many eigenvalues. The remaining assertions follow by applying Lemma 3.6. Rouchés Theorem and [18].

From formula (3.5) and Lemmas 3.3 and 3.5 we obtain Lemma 2.6 


\section{SPECTRAL MAPPING THEOREM}

We prove Theorem 2.1] For this we note some observations.

Theorem 4.1 (Spectral inclusion [1, Thm. 2.6, p.25]). $\forall t \geq 0: e^{t \sigma(A)} \subset \sigma\left(e^{A t}\right)$.

Theorem 4.2 (Gearhart-Prüss spectral mapping theorem [10, 11]). Let $\left(e^{A t}\right)_{t \geq 0}$ be a $C_{0}$ semigroup generated by $A$ in a Hilbert space. Then $e^{\lambda t} \in \rho\left(e^{A t}\right)$ iff

$$
\left\{\lambda+i 2 \pi t^{-1} z \mid z \in \mathbb{Z}\right\} \subset \rho(A) \quad \text { and } \sup _{z \in \mathbb{Z}}\left\|\left(\lambda+i 2 \pi t^{-1} z-A\right)^{-1}\right\|<\infty .
$$

Proof of Theorem 2.1. Theorem 4.1 yields $\sigma\left(e^{A t}\right) \backslash\{0\} \supset \overline{e^{\sigma(A) t}} \backslash\{0\}$. Hence it remains to show $\sigma\left(e^{A t}\right) \backslash\{0\} \subset \overline{e^{\sigma(A) t}} \backslash\{0\}$. Suppose $e^{\lambda t} \notin \overline{e^{\sigma(A) t}}$. Then Theorem4.1 implies that there exists a $\delta>0$ so that

$$
U_{\delta}:=\bigcup_{z \in \mathbb{Z}} B_{\delta}\left(\lambda+i 2 \pi t^{-1} z\right) \subset \rho(A) .
$$

There exists $d>0$ so that for all $\lambda \in U_{\frac{\delta}{2}}$ with $|\mathfrak{I m} \lambda| \geq d$ we have $h_{0}(\lambda) \neq 0$. If that is false, then we get a contradiction to (4.1) that $h$ has a zero on $U_{\delta}$. Indeed, suppose the contrary. Then there exists a sequence $\left(\lambda_{m}\right)_{m \in \mathbb{N}}$ in $U_{\frac{\delta}{2}}$ with $\left|\mathfrak{I m} \lambda_{m}\right| \rightarrow_{m \rightarrow \infty} \infty$ and $h_{0}\left(\lambda_{m}\right)=0$. Define $h_{0 m}(\lambda):=h_{0}\left(\lambda+\lambda_{m}\right), \tilde{h}_{m}(\lambda):=\tilde{h}\left(\lambda+\lambda_{m}\right)$. Since $h_{0}$ is an exponential polynomial, the real parts of $\lambda_{m}$ are bounded and the circle $S^{1}$ is compact, we have that $h_{0 m}$ converges uniformly to an exponential polynomial $h_{*}(\lambda)$ on each stripe $\mathbb{C}_{r}$ for any given $r>0$ (compare with the proof of Lemma 3.6). Moreover, estimate (2.2) yields that $\tilde{h}_{m}$ converges locally uniformly to $h_{*}$. Since $h_{*}(0)=0$ Hurwitz' theorem implies that for any $\epsilon>0$ there exists $\lambda_{\epsilon} \in \mathbb{C},\left|\lambda_{\epsilon}\right|<$ $\epsilon$, and $m \in \mathbb{N}$ so that $\tilde{h}\left(\lambda_{\epsilon}+\lambda_{m}\right)=\tilde{h}_{m}\left(\lambda_{\epsilon}\right)=0$. By choosing $\epsilon$ sufficiently small we get $\lambda_{\epsilon}+\lambda_{m} \in U_{\delta}$ with $h\left(\lambda_{\epsilon}+\lambda_{m}\right)=\tilde{h}\left(\lambda_{\epsilon}+\lambda_{m}\right)=0$. Hence, using Lemma 3.6 it follows that there exists $d>0$ so that $\inf _{\lambda \in U_{\frac{\delta}{4}},|\mathfrak{I m} \lambda|>2 d}\left|h_{0}(\lambda)\right|>0$. By applying Lemma 2.6 the resolvent is bounded on $U_{\frac{\delta}{4}}$. Theorem 4.2 yields that $e^{\lambda t} \in \rho\left(e^{A t}\right)$.

\section{ACKNOWLEDGMENTS}

Parts of the results are contained in the thesis [14] of the author supervised by L. Recke. The author would like to thank L. Recke, A. Mielke, K. Lu, K. Schneider and Y. Latushkin for helpful comments and support.

\section{REFERENCES}

1. Y. Latushkin and C. Chicone, Evolution Semigroups in Dynamical Systems and Differential Equations, Math. Surv. Monogr., Vol. 70, AMS, Providence, 1999. MR1707332(2001e:47068)

2. M. Renardy, Spectrally determined growth is generic, Proc. Amer. Math. Soc., 124 (1996), 2451-2453. MR1328372 (96j:47032)

3. - On the linear stability of hyperbolic PDEs and viscoelastic flows, Z. Angew. Math. Phys. (ZAMP), 45 (1994). 854-865. MR.1306936 (95i:35195)

4. H. Koch and D. Tataru, On the spectrum of hyperbolic semigroups, Comm. Partial Differential Equations, 20 (1994) 901-937. MR1326911 (97k:35150)

5. A. Vanderbauwhede and G. Iooss, Center manifold theory in infinite dimensions, Dynamics Reported, Vol. 1, new series (C. K. R. T. Jones, U. Kirchgraber, and H.-O. Walther editors), Springer, Heidelberg, 1992, pp. 123-163. MR1153030 (93f:58174) 
6. P. W. Bates and C. K. R. T. Jones, Invariant manifolds for semilinear partial differential equations, Dynamics Reported, Vol. 2, old series (U. Kirchgraber and H.-O. Walther editors), John Wiley \& Sons, 1989, pp. 1-38. MR1000974 (90g:58017)

7. P. W. Bates, K. Lu and C. Zeng, Existence and persistence of invariant manifolds for semiflows in Banach spaces, Mem. Amer. Math. Soc., 645 (1998), 129 pp. MR.1445489(99b:58210)

8. P. W. Bates and K. Lu and C. Zeng, Persistence of overflowing manifolds for semiflow, Comm. Pure Appl. Math., Vol. LII (1999), 983-1046. MR.1686965 (2000f:37116)

9. F. Gesztesy, C. K. R. T. Jones, Y. Latushkin, and M. Stanislavova, A spectral mapping theorem and invariant manifolds for nonlinear Schrödinger equations, Indiana Univ. Math. J., 49 (2000), 221-243. MR.1777032 (2001g:37144)

10. L. Gearhart, Spectral theory for contraction semigroups in Hilbert space, Trans. Amer. Math. Soc., 236 (1978), 385-394. MR0461206 (57:1191)

11. J. Prüss, On the spectrum of $C_{0}$ semigroups, Trans. Amer. Math. Soc., 284 (1984), 847-857. MR743749 (85f:47044)

12. A. F. Neves, H. Ribeiro, and O. Lopes, On the spectrum of evolution operators generated by Hyperbolic Systems, J. Funct. Anal., 67 (1986) 320-344. MR845461 (87k:35155)

13. M. Renardy, On the type of certain $C_{0}$-semigroups, Comm. Partial Differential Equations, 18 (1993), 1299-1307. MR1233196 (94h:47080)

14. M. Lichtner, Exponential dichotomy and smooth invariant center manifolds for semilinear hyperbolic systems, $\mathrm{PhD}$ thesis, 2006, 167 pp.; http://dochost.rz.hu-berlin.de/ browsing/dissertationen/

15. _ Principle of linearized stability and center manifold theorem for semilinear hyperbolic systems, WIAS preprint No. 1155, 2006, 28 pp.

16. - Variation of constants formula for hyperbolic systems, WIAS preprint No. 1212, 2007, $19 \mathrm{pp}$.

17. M. Radziunas, Numerical bifurcation analysis of traveling wave model of multisection semiconductor lasers, Physica D, 213(1) (2006), 98-112. MR2186586 (2006k:82160)

18. X-B Lin and A. F. Neves, A multiplicity theorem for hyperbolic systems, J. Differential Equations, 76 (1988), 339-352. MR969429 (90d:47051)

19. Z. H. Luo, B. Z. Guo, and O. Morgul, Stability and Stabilization of Infinite Dimensional Systems with Applications, Springer, 1999. MR.1745384 (2001j:93001)

20. B. Z. Guo and G. Q. Xu, On Basis property of a hyperbolic system with dynamic boundary condition, Differential Integral Equations, 18 (2005) no. 1, 35-60.

Weierstrass Institute for Applied Analysis and Stochastics, Mohrenstr. 39, 10117 BerLin, Germany

E-mail address: lichtner@wias-berlin.de 